\title{
Room temperature scanning Hall probe microscopy using GaAs/AlGaAs and Bi micro-hall probes
}

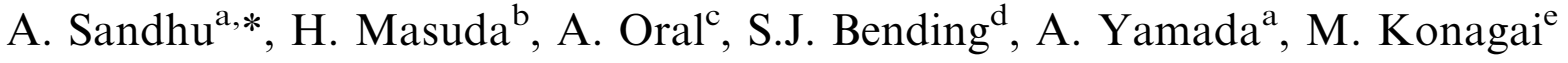 \\ ${ }^{a}$ Research Center for Quantum Effect Electronics, Tokyo Institute of Technology, 2-12-1 O-okayama, Meguro-ku, Tokyo 152-8552, \\ Japan \\ ${ }^{\mathrm{b}}$ Toei Kogyo Ltd., 8-13-1, Tadao, Machida 194-0035, Japan \\ ${ }^{\mathrm{c}}$ Department of Physics, Bilkent University, Ankara 06533, Turkey \\ ${ }^{\mathrm{d}}$ Department of Physics, University of Bath, BA 2 7AY, UK \\ ${ }^{\mathrm{e}}$ Department of Physical Electronics, Tokyo Institute of Technology, 2-12-1 O-Okayama, Meguro-ku, Tokyo 152-8552, Japan
}

Received 29 May 2001; received in revised form 20 November 2001

\begin{abstract}
A room temperature scanning Hall probe microscope system utilizing GaAs/AlGaAs and bismuth micro-Hall probes was used for magnetic imaging of ferromagnetic domain structures on the surfaces of crystalline thin film garnets and permanent magnets. The Bi micro-Hall probes had dimensions ranging between 0.25 and $2.8 \mu \mathrm{m}^{2}$ and were fabricated using a combination of optical lithography and focused ion beam milling. The use of bismuth was found to overcome surface depletion effects associated with semiconducting micro-Hall probes. Our experiments demonstrated that $\mathrm{Bi}$ is a practical choice of material for fabricating sub-micron sized Hall sensors. (C) 2002 Elsevier Science B.V. All rights reserved.
\end{abstract}

Keywords: Ferromagnetic domains; Hall sensors; Nanotechnology; Bismuth; GaAs; Permanent magnetics; Garnets

\section{Introduction}

We have previously reported on the development and use of a room temperature scanning Hall probe microscope system (RT-SHPM) for directly imaging magnetic domains on the surfaces of ferromagnetic materials where a $\sim 1.2 \mu \mathrm{m}^{2}$ sized GaAs/AlGaAs two-dimensional electron gas (2DEG) heterostructure micro-Hall probe (GaAs/AlGaAs micro-HP) was used as the sensing

\footnotetext{
*Corresponding author. Permanent address. Department of Electrical and Electronic Engineering, Tokai University, Hiratsuka, 1117 Kitakaname, Kanagawa 259-1292, Japan. Tel.: +81-463-58-9203; fax: +81-463-58-9203.

E-mail address: sandhu@keyaki.cc.u-tokai.ac.jp (A. Sandhu).
}

element [1]. A combination of the dimensions of the active area ('cross region') of the HP and distance from the sample surface determines the ultimate spatial resolution of a scan. At room temperature it is relatively easy to design piezoelectric actuator assemblies in order to position a Hall probe to less than $50 \mathrm{~nm}$ from the surface but it is extremely difficult to reduce the active area of GaAs/AlGaAs HPs to less than $\sim 1 \mu \mathrm{m}^{2}$ due to surface charge depletion effects that limit the maximum Hall probe drive current and hence the magnetic field sensitivity of the Hall device [2]. Further, the high series resistance of GaAs/ AlGaAs 2DEG sensors leads to high thermal noise (Johnson noise) which in turn degrades the signal-to-noise ratio at room temperature. An 
alternative material for fabricating Hall probes is bismuth (Bi), a semimetal with a carrier concentration of five orders of magnitude lower than metals and the advantage over semiconductors of negligible surface charge depletion effects [3].

In this paper, we describe the results of a study to experimentally determine the practical limits of $\mathrm{GaAs} / \mathrm{AlGaAs}$ and Bi micro-Hall probes when used as magnetic field sensing elements in the RT-SHPM. We were unable to fabricate functional GaAs/ AlGaAs Hall probes with dimensions less than $\sim 1.0 \mu \mathrm{m}^{2}$. However, Bi Hall probes with dimensions as small as $0.25 \mu \mathrm{m} \times 0.25 \mu \mathrm{m}$ were fabricated by focused ion beam (FIB) milling and successfully used for RT-SHPM magnetic imaging of garnets. Bi is demonstrated to be a practical alternative for the fabrication of sub-micron Hall probes for RTSHPM imaging of magnetic domain structures.

\section{Experimental}

Fig. 1 shows the main components of the RTSHPM used in this study. The system was specifically designed for operation at room temperature for scans up to $50 \mu \mathrm{m} \times 50 \mu \mathrm{m}$. The $\mathrm{GaAs} / \mathrm{AlGaAs}$ and $\mathrm{Bi}$ micro-Hall probe sensors were mounted onto the piezoelectric scanning tube (PZT) at a tilt angle of $1.5^{\circ}$ with respect to the sample. The GaAs/AlGaAs 2DEG (electron density and mobility of $2 \times 10^{11} \mathrm{~cm}^{-2}$ and $400,000 \mathrm{~cm}^{2} / \mathrm{Vs}$, respectively, at $4.2 \mathrm{~K}$ ) and $\mathrm{Bi}$ micro-HPs were fabricated by conventional photolithography as previously described $[1,4]$. Fig. 2(a) shows a typical Bi micro-HP, where the integrated STM-tip was used for precise vertical positioning as well as topographic imaging during RT-SHPM measurements. The physical sizes of the GaAs/ AlGaAs were $\sim 1.2 \mu \mathrm{m}^{2}$ and those of Bi microHPs ranged between 0.25 and $2.8 \mu \mathrm{m}^{2}$. The active area of the GaAs/AlGaAs was $\sim 0.8 \mu \mathrm{m}^{2}$ due to surface depletion effects.

Fig. 2(b) is a scanning electron microscope image of a $0.25 \mu \mathrm{m}^{2}$ Bi micro-HP produced by FIB milling of the larger $\mathrm{Bi}$ micro-Hall probe (Fig. 2(a)) using a Hitachi FB-2000A FIB system by scanning gallium ions (focused to $10 \mathrm{~nm}$ ) over the cross region. Typical FIB milling employed ion

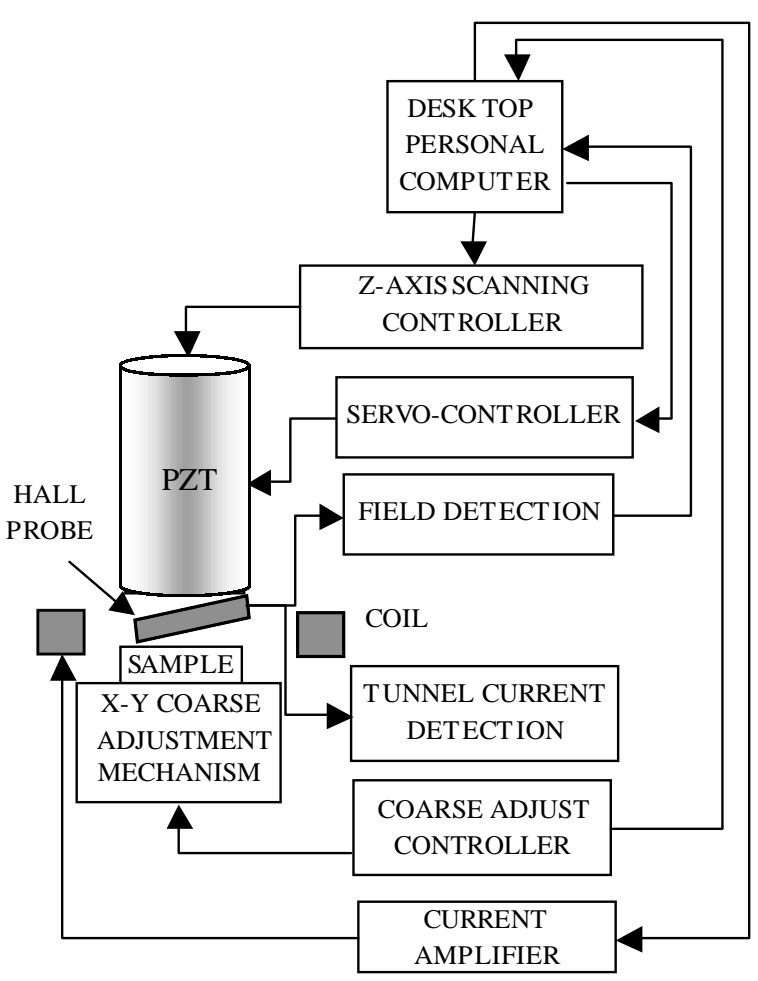

Fig. 1. Main components of the RT-SHPM system.

currents and voltages of $15 \mathrm{~A} / \mathrm{cm}^{2}$ and $30 \mathrm{kV}$, respectively. The scanning ion microscope mode (SIM) of the FIB system was used for monitoring the device structure before and after milling.

Magnetic imaging was carried out by scanning the HP over a ferromagnetic sample surface while simultaneously monitoring changes in Hall voltage that arise due to fluctuations of the perpendicular component of the magnetic field emanating from the surface. The black and white regions of the RT-SHPM images represent magnetic domains with magnetizations into and out of the plane of the paper. The results described in this paper were obtained with the Hall probe at a height of $0.29 \mu \mathrm{m}$ above the surface of the samples. A unique set of program routines using the interactive data language (IDL) were developed for colored three-dimensional visualization and animation of the raw black and white image data. ${ }^{1}$

\footnotetext{
${ }^{1}$ Research Systems Inc., 4990 Pearl East Circle, Boulder, CO, USA.
} 


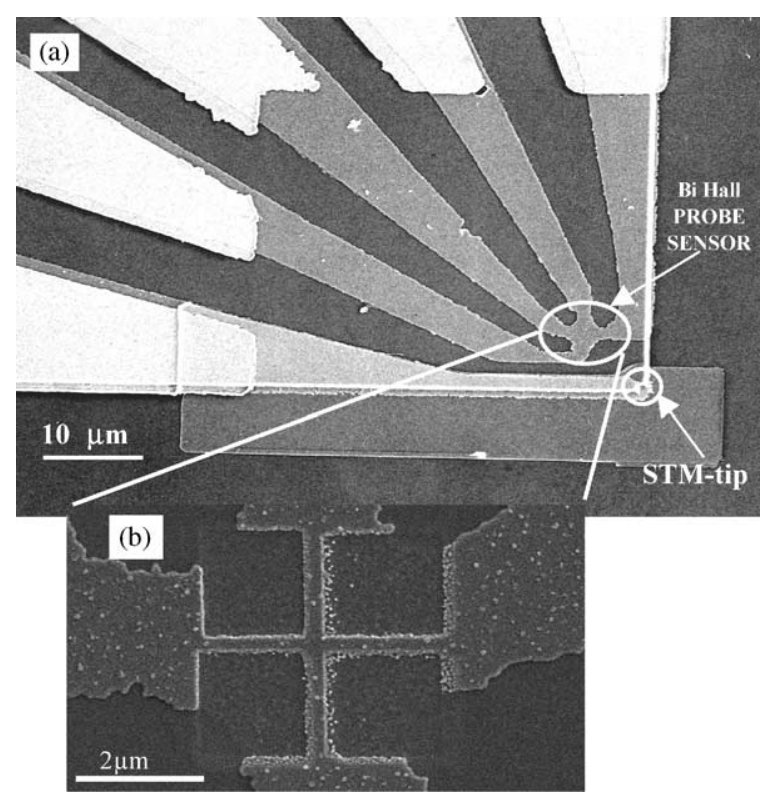

Fig. 2. (a) Scanning electron microscope image of a typical Bi micro-Hall probe and STM-tip. (b) Scanning electron microscope image of a $0.25 \mu \mathrm{m} \times 0.25 \mu \mathrm{m}$ Bi Hall probe.

\section{Results and discussion}

The Hall coefficient $\left(R_{\mathrm{H}}\right)$ and series resistance $\left(R_{\mathrm{S}}\right)$ of a $2.8 \mu^{2}$ Bi micro-HP were measured to be $3.3 \times 10^{-4} \Omega / \mathrm{G}$ and $2 \mathrm{k} \Omega$, respectively. The magnetic field resolution, that is, signal-to-noise ratio $(S / N)$ of a Hall probe can be defined as

$\frac{S}{N}=\frac{\left(I_{\mathrm{H}} R_{\mathrm{H}} B\right)}{\sqrt{4 k_{\mathrm{B}} T R_{\mathrm{s}} \Delta f}}$

where $I_{\mathrm{H}}$ is the Hall probe drive current, $B$ is the magnetic induction being measured, $\Delta f$ is the measurement band width, $k_{\mathrm{B}}$ is Boltzmann's constant, and $T$ is the measurement temperature. Here, $\sqrt{4 k_{\mathrm{B}} T R_{\mathrm{S}} \Delta f}$ is the Johnson noise and main component of the measurement noise and $\left(I_{\mathrm{H}} R_{\mathrm{H}} B\right)$ the Hall voltage at a given drive Hall current and applied field. Using a spectrum analyzer, the Johnson noise of the Bi-HP was determined to be $100 \mathrm{nV} / \sqrt{ } \mathrm{Hz}$ at a maximum driving Hall current of $800 \mu \mathrm{A}$ and the resulting $S / N$ ratio was determined to be $0.38 \mathrm{G} / \sqrt{ } \mathrm{Hz}$. A small $1 / f$ noise component was also observed.
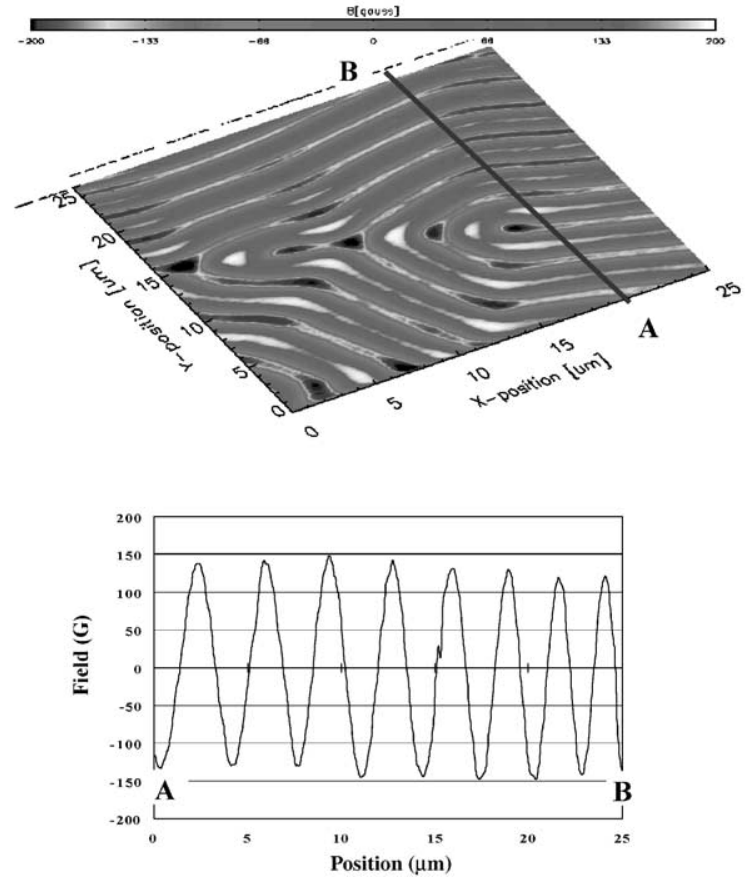

Fig. 3. A $25 \mu \mathrm{m} \times 25 \mu \mathrm{m}$ image of a $5.5 \mu \mathrm{m}$ thick crystalline Bisubstituted iron garnet thin film measured using the GaAs/ AlGaAs HP with a drive current of $2 \mu \mathrm{A}$. The graph is the variation of magnetic field along the line drawn on the image.

Similar noise measurements were carried out on a $\sim 1.2 \mu \mathrm{m}^{2} \mathrm{GaAs} / \mathrm{AlGaAs}$ micro-Hall probe with $R_{\mathrm{H}}$ and $R_{\mathrm{s}}$ of $0.26 \Omega / \mathrm{G}$ and $70 \mathrm{k} \Omega$, respectively. The Johnson noise of this GaAs/AlGaAs HP was $316 \mathrm{nV} / \sqrt{ } \mathrm{Hz}$ at a maximum driving Hall current of $2 \mu \mathrm{A}$ Hall and the resulting $S / N$ ratio was $0.41 \mathrm{G} /$ $\sqrt{H z}$. Again a $1 / f$ noise component was also observed.

A selection of representative RT-SHPM images obtained using the GaAs/AlGaAs and Bi HPs will now be described. No correlation was observed between the magnetic and topographical images of any of the materials studied.

Fig. 3 is a $25 \mu \mathrm{m} \times 25 \mu \mathrm{m}$ image of a $5.5 \mu \mathrm{m}$ thick crystalline Bi-substituted iron garnet thin film (Fegarnet) measured using the GaAs/AlGaAs micro$\mathrm{HP}$ at a drive current of $2 \mu \mathrm{A}$. The graph is the variation of magnetic field along the line drawn on the image. Fig. 4(a) is a $50 \mu \mathrm{m} \times 50 \mu \mathrm{m}$ image of a $360 \mu \mathrm{m}$ thick Fe-garnet, again measured using the GaAs/AlGaAs micro-HP and Fig. 4(b) is a three- 

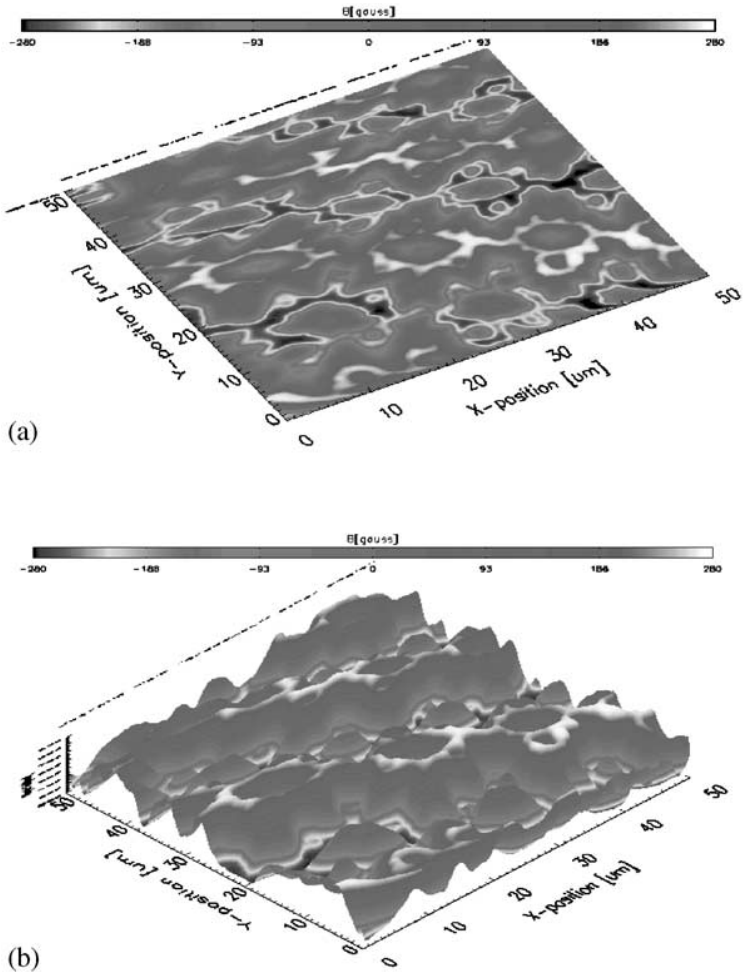

Fig. 4. (a) A $50 \mu \mathrm{m} \times 50 \mu \mathrm{m}$ image of a $360 \mu \mathrm{m}$ thick bismuthsubstituted iron garnet thin film measured using the GaAs/ AlGaAs HP. (b) A three-dimensional visualization of the same measurement.

dimensional visualization of the same measurement. The surface magnetic field is seen to vary in between \pm 280 G. Fig. 5 is a $50 \mu \mathrm{m} \times 50 \mu \mathrm{m} \mathrm{GaAs} /$ AlGaAs micro-HP image of the polished surface of a SmCo permanent magnet in its remanent state. Domains are clearly visible with field variations in between $\pm 4090 \mathrm{G}$.

Figs. 6(a) and (b) are raw $25 \mu \mathrm{m} \times 25 \mu \mathrm{m}$ RTSHPM images measured using $\sim 2.8 \mu \mathrm{m}^{2}$ Bi microHP (drive current of $400 \mu \mathrm{A}$ ), showing the changes in the stripe domain structure of the $5.5 \mu \mathrm{m}$ thick Fe-garnet under perpendicular external bias fields of 1021 and $1131 \mathrm{Oe}$, respectively. The variation of the magnetic field along the lines drawn on the images is also shown. These results show one stage of the process of configurational hysteresis of domain structures in low coercivity films that exhibit strong perpendicular magnetic anisotropy [1]. Fig. 7 is a RT-SHPM Bi micro-HP image of

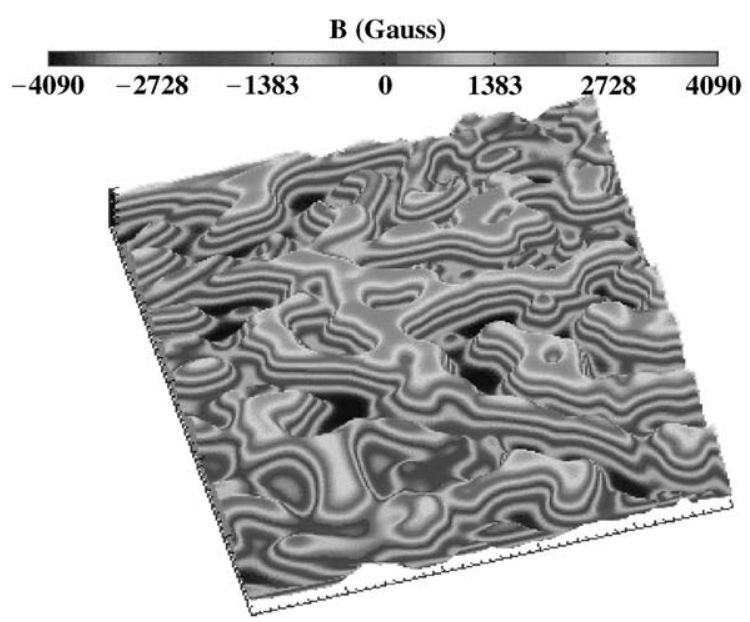

Fig. 5. A $50 \mu \mathrm{m} \times 50 \mu \mathrm{m} \mathrm{GaAs} / \mathrm{AlGaAs}$ HP image of a SmCo permanent magnet in its remanent state. The measured surface field varies in between $\pm 4090 \mathrm{G}$.
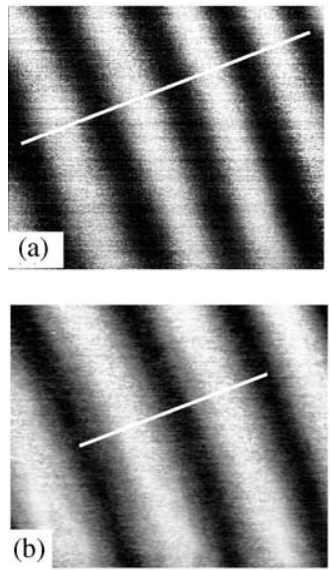
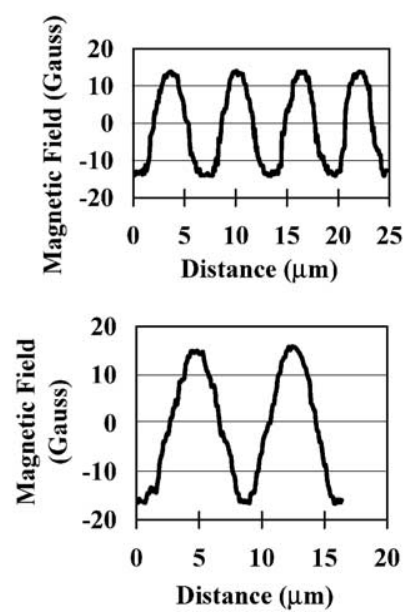

Fig. 6. Bi HP images of $25 \mu \mathrm{m} \times 25 \mu \mathrm{m}$ showing changes in the stripe domain structure of a $5.5-\mu \mathrm{m}$ thick crystalline Bisubstituted iron garnet thin film under perpendicular external bias fields of 1021 Oe (a) and 1131 Oe (b). The graphs are the field variations along the lines indicated.

the polished surface of a $400 \mu \mathrm{m}$ thick demagnetized strontium ferrite permanent magnet showing microscopic domain structures with fields varying as shown in the accompanying graph.

Fig. 8 shows a typical $20 \mu \mathrm{m} \times 20 \mu \mathrm{m}$ RT-SHPM magnetic image of the $5.5-\mu \mathrm{m}$ thick bismuth- 

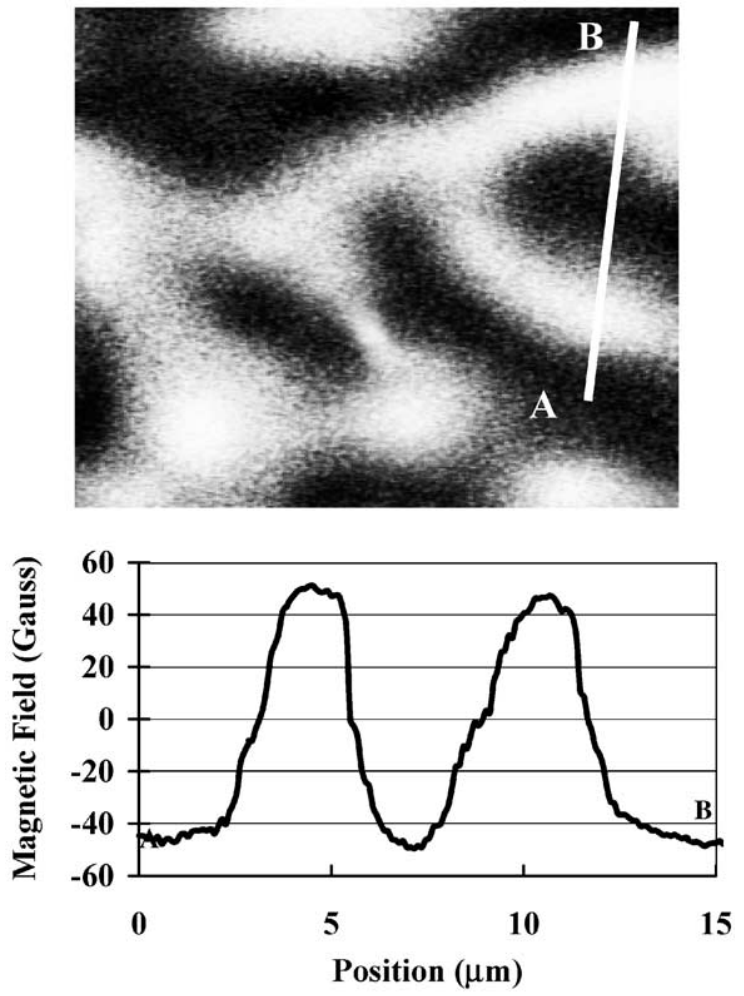

Fig. 7. A $25 \mu \mathrm{m} \times 25 \mu \mathrm{m}$ Bi-HP image of the domain structure observed on the surface of a demagnetized strontium ferrite permanent magnet. The graph shows the field variation along the line $\mathrm{A}-\mathrm{B}$.

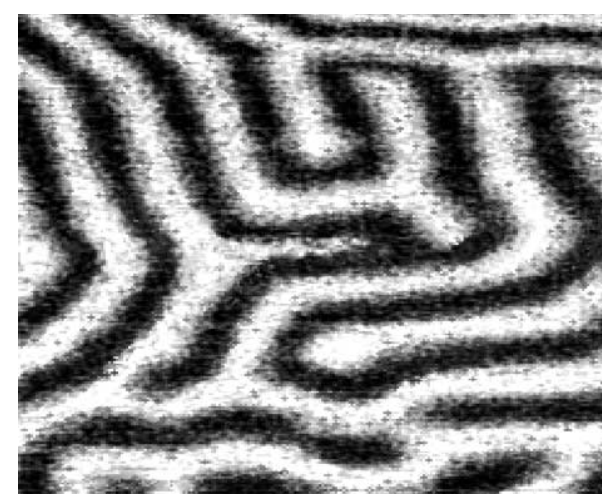

Fig. 8. A $20 \mu \mathrm{m} \times 20 \mu \mathrm{m}$ RT-SHPM image of a $5.5-\mu \mathrm{m}$ thick bismuth-substituted iron garnet thin film measured using a $0.25 \mu \mathrm{m} \times 0.25 \mu \mathrm{m}$ Bi Hall probe.

substituted iron garnet thin film obtained using the $0.25 \mu \mathrm{m}^{2} \mathrm{Bi}$ Hall probe with the following electrical characteristics at a driving current of $40 \mu \mathrm{A}$ : series resistance of $4.5 \mathrm{k} \Omega$; Johnson noise of $110 \mathrm{nV} / \sqrt{ } \mathrm{Hz}$; and magnetic field sensitivity of $7.2 \mathrm{G} / \sqrt{ } \mathrm{Hz}$. The measured fields vary in between $\pm 165 \mathrm{G}$ from black to white.

We also attempted to fabricate GaAs/AlGaAs HPs with 'cross-regions' having dimensions of $\sim 0.25 \mu \mathrm{m}^{2}$ using electron beam lithography but found such probes to have extremely high series resistances and it was not possible to pass a drive current through the devices at room temperature. However, as described above, the Bi probes were conducting even with dimensions as small as $0.25 \mu \mathrm{m}^{2}$ and in spite of a smaller Hall coefficient $\left(\mathrm{Bi}=3.3 \times 10^{-4} \Omega / \mathrm{G} ; \quad \mathrm{GaAs} / \mathrm{AlGaAs}=0.26 \Omega / \mathrm{G}\right)$, it was possible to use them for magnetic imaging. The use of $\mathrm{Bi}$ for fabricating Hall probes is an important advance for room temperature magnetic imaging of ferromagnetic surfaces on the nanometer scale.

\section{Summary}

Bismuth is a promising alternative to $\mathrm{GaAs} /$ $\mathrm{AlGaAs}$ for the fabrication of nanometer sized Hall probes for the magnetic imaging of ferromagnetic domain structures by room temperature scanning Hall probe microscopy.

\section{Acknowledgements}

This work was partly funded by the Japanese Ministry of Education, Culture, Sports and Science and Technology (Grant in Aid \#13650354).

\section{References}

[1] A. Sandhu, H. Masuda, A. Oral, S.J. Bending, Proceedings of the Eighth International Conference on Ferrites, Kyoto, September 18-21, 2000.

[2] G. Jung, M. Ocio, Y. Paltiel, H. Shtrikman, E. Zeldov, Appl. Phys. Lett. 78 (2001) 359.

[3] J.A. van Hulst, H.M. Jaeger, S. Radelaar, Phys. Rev. B. 52 (1995) 5953.

[4] A. Sandhu, H. Masuda, A. Oral, S.J. Bending, Jpn. J. Appl. Phys. 40 (5B) (2001) L524. 